\title{
Energy metabolism determines the sensitivity of human hepatocellular carcinoma cells to mitochondrial inhibitors and biguanide drugs
}

\author{
CHIA-CHI HSU ${ }^{1}$, LING-CHIA WU ${ }^{1}$, CHENG-YUAN HSIA ${ }^{2}$, PEN-HUI YIN ${ }^{3}$, \\ CHIN-WEN CHI ${ }^{1,3}$, TIEN-SHUN YEH ${ }^{4}$ and HSIN-CHEN LEE ${ }^{1}$ \\ ${ }^{1}$ Department and Institute of Pharmacology, School of Medicine, National Yang-Ming University, Taipei 112; \\ Departments of ${ }^{2}$ Surgery and ${ }^{3}$ Medical Research, Taipei Veterans General Hospital, Taipei; \\ ${ }^{4}$ Department of Anatomy and Cell Biology, School of Medicine, \\ National Yang-Ming University, Taipei 112, Taiwan, R.O.C.
}

Received April 9, 2015; Accepted June 15, 2015

DOI: $10.3892 /$ or.2015.4092

\begin{abstract}
Human hepatocellular carcinoma (HCC) is one of the most common malignancies worldwide particularly in Asia. Deregulation of cellular energetics was recently included as one of the cancer hallmarks. Compounds that target the mitochondria in cancer cells were proposed to have therapeutic potential. Biguanide drugs which inhibit mitochondrial complex I and repress mTOR signaling are clinically used to treat type 2 diabetes mellitus patients (T2DM) and were recently found to reduce the risk of HCC in T2DM patients. However, whether alteration of energy metabolism is involved in regulating the sensitivity of HCC to biguanide drugs is still unclear. In the present study, we treated four HCC cell lines with mitochondrial inhibitors (rotenone and oligomycin) and biguanide drugs (metformin and phenformin), and found that the HCC cells which had a higher mitochondrial respiration
\end{abstract}

Correspondence to: Dr Hsin-Chen Lee, Department and Institute of Pharmacology, School of Medicine, National Yang-Ming University, 115 Li-Nong Street, Section 2, Taipei 112, Taiwan, R.O.C.

E-mail: hclee2@ym.edu.tw

Abbreviations: 4E-BPs, eIF4E-binding proteins; AMPK, AMPactivated protein kinase; FCCP, carbonyl cyanide 4-(trifluoromethoxy) phenylhydrazone; DMEM, Dulbecco's modified Eagle's medium; ECAR, extracellular acidification rate; HA22T, HA22T/VGH; HCC, hepatocellular carcinoma; HIF-1 $\alpha$, hypoxia inducible factor- $1 \alpha$; LDHA, lactate dehydrogenase A; LKB1, liver kinase B1; mTOR, mammalian target of rapamycin; OCR, oxygen consumption rate; OXPHOS, oxidative phosphorylation; PDK, pyruvate dehydrogenase kinase; PI, propidium iodide; PKM2, pyruvate kinase M2; RTK, receptor tyrosine kinase; SRB, sulforhodamine B; T2DM, type 2 diabetes mellitus; TACE, transarterial chemoembolization

Key words: hepatocellular carcinoma, biguanides, mitochondrial inhibitors, AMPK-mTOR, transarterial chemoembolization rate were more sensitive to these treatments; whereas the HCC cells which exhibited higher glycolysis were more resistant. When glucose was replaced by galactose in the medium, the altered energy metabolism from glycolysis to mitochondrial respiration in the HCC cells enhanced the cellular sensitivity to mitochondrial inhibitors and biguanides. The energy metabolism change enhanced AMP-activated protein kinase (AMPK) activation, mTOR repression and downregulation of cyclin D1 and Mcl-1 in response to the mitochondrial inhibitors and biguanides. In conclusion, our results suggest that increased mitochondrial oxidative metabolism upregulates the sensitivity of HCC to biguanide drugs. Enhancing the mitochondrial oxidative metabolism in combination with biguanide drugs may be a therapeutic strategy for HCC.

\section{Introduction}

Hepatocellular carcinoma (HCC) is one of the most common malignancies worldwide, particularly in Asia. The current treatments for HCC patients include surgical resection, local ethanol injection, transarterial chemoembolization (TACE), liver transplant, radiation therapy, chemotherapy and target therapy (1-3). However, more than two-thirds of HCC patients are not indicated for surgical resection due to large tumor size, poor hepatic function or metastasis (4). Moreover, HCC seems to be resistant to most chemotherapies and radiation therapy (2). Therefore, it is urgent to improve the drug sensitivity of HCC and to develop new strategies to treat HCC patients.

Most cancer cells prefer to use glycolysis rather than utilize oxidative phosphorylation (OXPHOS) for glucose metabolism even in oxygen-rich conditions, this is termed aerobic glycolysis or the 'Warburg effect' (5). Deregulated cellular energetics was recently included as one new cancer hallmark (6). This is due to the fast progress in understanding various molecular mechanisms of the Warburg effect in cancer cells. These mechanisms include oncogenic activation, inhibition of tumor-suppressor genes or mitochondrial dysfunction due to nuclear/mitochondrial DNA mutations (7-9). These metabolic features facilitate the survival, proliferation and 
metastasis of cancer cells (10). Therefore, targeting energy metabolism in cancer cells has become an important focus of cancer therapy (11).

Mammalian target of rapamycin (mTOR) is a serine/threonine kinase which modulates numerous cellular functions including cell growth, migration and protein translation $(12,13)$. Highly activated mTOR signaling due to mutations of receptor tyrosine kinase (RTK), amplification of AKT or loss of PTEN has been observed in several types of cancer including HCC $(14,15)$. The $\mathrm{p} 70^{\mathrm{S} 6 \mathrm{~K}}$ and eIF4E-binding proteins (4E-BPs), which can be phosphorylated by the mTOR complex, promote protein synthesis for cell growth and survival $(12,16)$. AMP-activated protein kinase (AMPK) is an energy sensor and is involved in the regulation of mTOR signaling. During energy stress, AMPK is activated by its upstream liver kinase B1 (LKB1) and further suppresses mTOR signaling for cellular adaptation in a stress condition $(17,18)$. LKB1 is thought to be a tumor suppressor, and genetic loss of LKB1 is a frequent event in several types of cancer, including HCC (19-22). The loss of LKB1 expression contributes to the aberrant activation of mTOR signaling in cancer cells (23). Hence, identification of ways to reduce mTOR signaling is a therapeutic strategy against cancer.

Biguanide drugs, particularly metformin, are used to treat type 2 diabetic patients (24). Studies show that the biguanide drugs reduce the risk of $\mathrm{HCC}$ in type 2 diabetes mellitus patients (T2DM) and thus suggest that biguanide drugs can be used as adjuvant reagents for the treatment of HCC patients (25-29). The biguanides were found to inhibit Complex I of the mitochondrial respiratory chain (29), and to repress mTOR signaling through AMPK-dependent (30) and -independent pathways (31) in cellular experiments. However, it is unclear whether alteration of the cellular energy metabolism affects the sensitivity of HCC cells to biguanide drugs.

In HepG2 HCC cells, mitochondrial inhibitors have been shown to activate AMPK and repress mTOR signaling, which downregulates HIF-1 $\alpha$ protein expression (32). In the present study, we found that various HCC cell lines (Mahlavu, SK-HEP-1 and HA22T/VGH) exhibited resistance to mitochondrial inhibitors and biguanide drugs in the examined HCC cell lines. The role of energy metabolism in regulating the sensitivity of HCC cells to mitochondrial inhibitors and biguanides was further evaluated.

\section{Materials and methods}

Reagents and antibodies. Biguanide drugs including metformin hydrochloride (cat. no. PHR1084, purity $>99.9 \%$ ) and phenformin hydrochloride (cat. no. P7045, purity $>97 \%$ ), and mitochondrial inhibitors including oligomycin (cat. no. O4876, purity $>90 \%$ ) and rotenone (cat. no. R8875, purity $>95 \%$ ) were purchased from Sigma-Aldrich (St. Louis, MO, USA). Cell culture medium was purchased from Gibco (Grand Island, NY, USA). Fetal bovine serum (FBS), L-glutamine and non-essential amino acids were obtained from Biological Industries (Kibbutz Beit Haemek, Israel). The antibodies against 4E-BP-1, phospho4E-BP-1 (Thr70), ACC, phospho-ACC (Ser79), AMPK $\alpha$, phospho-AMPK $\alpha$ (Thr172), cyclin D1, LKB1, phospho-LKB1 (Ser428), Mcl-1, p70S6 kinase, phospho-p70S6 kinase (Thr389), raptor and phosphor-raptor (Ser792) were purchased from
Cell Signaling Technology (Beverly, MA, USA). Aprotinin, EGTA, FCCP, $\mathrm{Na}_{3} \mathrm{VO}_{4}$, PMSF, D-glucose, D-galactose and the antibody against $\alpha$-tubulin were purchased from SigmaAldrich. AICAR-riboside (cat. no. 123040, purity >99.6\%) was purchased from Merck Millipore (West Point, PA, USA).

Cell cultures. Human hepatoma cells (HepG2, Mahlavu, SK-HEP-1 and HA22T/VGH) were cultured in Dulbecco's modified Eagle's medium (DMEM) supplemented with $10 \% \mathrm{FBS}, 2 \mathrm{mmol} / \mathrm{l} \mathrm{L}$-glutamine, $10 \mathrm{mmol} / 1 \mathrm{non}$-essential amino acids, $100 \mathrm{U} / \mathrm{ml}$ of penicillin and $0.1 \mathrm{mg} / \mathrm{ml}$ of streptomycin at $37^{\circ} \mathrm{C}$ in a humidified $5 \% \mathrm{CO}_{2}$ incubator.

For changing cellular energy metabolism from glycolysis to mitochondrial OXPHOS, the hepatoma cells were grown in medium in which glucose was replaced with galactose according to a previous study (33). Briefly, the hepatoma cells were cultured in D-glucose-free DMEM supplemented with $25 \mathrm{mM}$ D-galactose, $10 \% \mathrm{FBS}, 2 \mathrm{mmol} / 1 \mathrm{~L}$-glutamine, $10 \mathrm{mmol} / \mathrm{l}$ non-essential amino acids, $100 \mu \mathrm{g} / \mathrm{ml}$ pyruvate, $100 \mathrm{U} / \mathrm{ml}$ of penicillin, and $0.1 \mathrm{mg} / \mathrm{ml}$ of streptomycin at $37^{\circ} \mathrm{C}$ in a humidified $5 \% \mathrm{CO}_{2}$ incubator.

Cell viability analysis. Cell viability was determined using sulforhodamine B (SRB) assay. The cells $\left(5 \times 10^{3}\right)$ were seeded on 96-well plates overnight before each experiment. After treatment with mitochondrial inhibitors or biguanide drugs for $24 \mathrm{~h}$, the cells were fixed with $10 \%$ ice-cold trichloroacetic acid (TCA) (Sigma-Aldrich) at $4^{\circ} \mathrm{C}$ for $1 \mathrm{~h}$, rinsed four times with distilled water and air dried. The cells were then stained with $0.057 \%$ SRB (Sigma-Aldrich) in $1 \%$ acetic acid for $30 \mathrm{~min}$ at room temperature. After rinsing four times with $1 \%$ acetic acid and air dried, $50 \mu \mathrm{l}$ of $10 \mathrm{mM}$ Tris-base ( $\mathrm{pH} 10.5)$ was added into each well for $30 \mathrm{~min}$. The colorimetric level was read by a microplate reader (Tecan) at $510 \mathrm{~nm}$.

Western blot analysis. Whole cell extracts were prepared using radioimmunoprecipitation assay (RIPA) buffer $(150 \mathrm{mM}$ $\mathrm{NaCl}, 50 \mathrm{mM}$ Tris- $\mathrm{HCl}, 0.1 \%$ SDS, $0.5 \%$ sodium deoxycholate, $0.1 \%$ Triton X-100) plus $10 \mu \mathrm{g} / \mathrm{ml}$ aprotinin, 2 mM EGTA, $2 \mathrm{mM} \mathrm{Na}_{3} \mathrm{VO}_{4}$ and $1 \mathrm{mM}$ PMSF. The protein concentrations were determined using the Bradford assay (Sigma-Aldrich) and samples were diluted in 5X Laemmli buffer $[300 \mathrm{mM}$ Tris-HCl pH 6.8, 10\% SDS (w/v), 5\%, 2-mercaptoethanol, $25 \%$ glycerol (v/v), $0.1 \%$ bromphenol blue (w/v)] and boiled for $5 \mathrm{~min}$. Proteins $(40 \mu \mathrm{g})$ were separated by $8-15 \%$ SDS-PAGE and transferred onto polyvinylidene fluoride (PVDF) membranes (Pall Life Sciences). Non-specific binding sites on the PVDF membranes were blocked with $5 \%$ non-fat milk in TBST (20 mM Tris-HCl, pH 7.6, 137 mM NaCl, 1\% Tween20). Membranes were then hybridized with primary antibodies overnight at $4^{\circ} \mathrm{C}$, followed by incubation with horseradish peroxidase (HRP)-conjugated secondary antibodies. The membranes were then developed using Immobilon Western Chemiluminescence HRP Substrates (Millipore). Images were captured by a Luminescence/Fluorescence Imaging System (GE Healthcare), and signal intensities were quantified using Multi Guage image analysis software (Fujifilm).

$O C R$ and ECAR analyses. The oxygen consumption rate (OCR) and extracellular acidification rate (ECAR) of the 
A

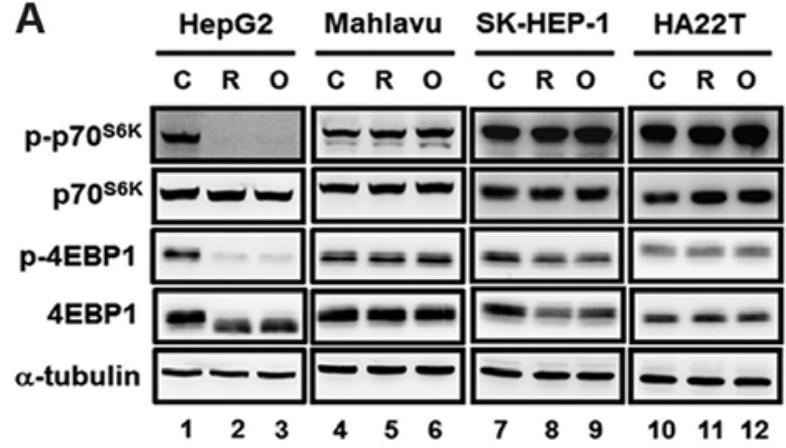

B

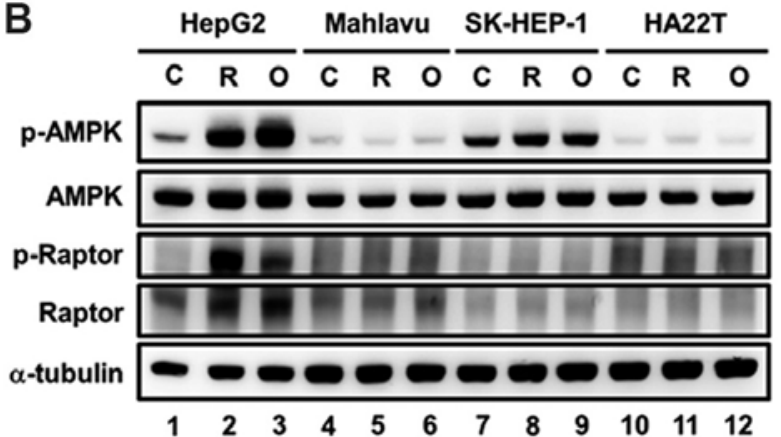

C

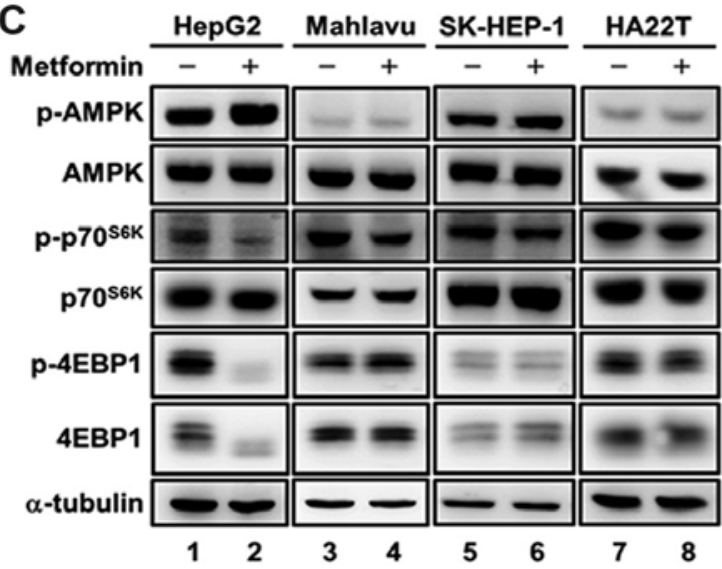

D

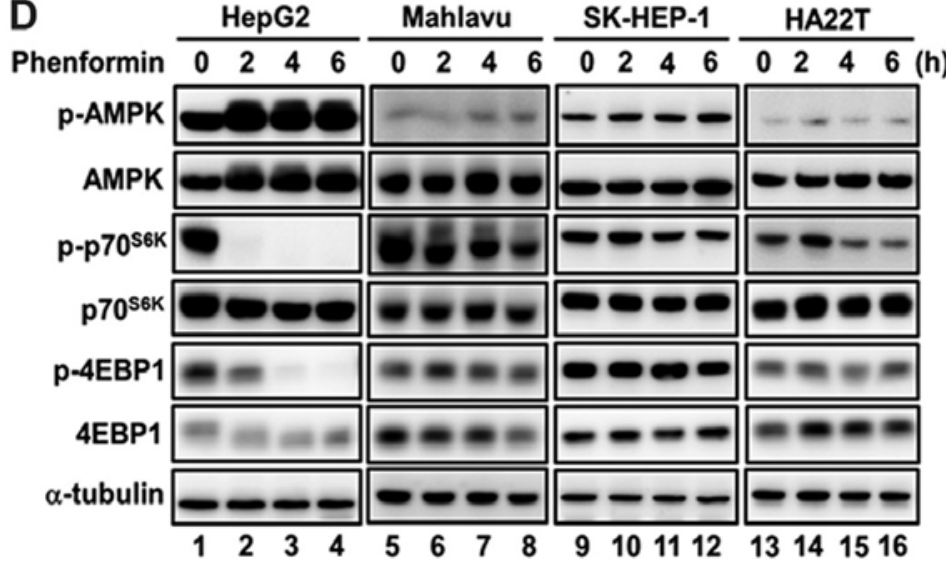

Figure 1. Effect of mitochondrial inhibitors and biguanides on the AMPK-mTOR signaling in HCC cells. HCC cell lines (HepG2, Mahlavu, SK-HEP-1 and HA22T/VGH) were treated with (A and B) mitochondrial inhibitors $[0.2 \mu \mathrm{M}$ rotenone $(\mathrm{R})$ and $1 \mu \mathrm{g} / \mathrm{ml}$ oligomycin $(\mathrm{O})]$ for $3 \mathrm{~h}$ or $(\mathrm{C}$ and $\mathrm{D})$ biguanide drugs $(5 \mathrm{mM}$ metformin for $9 \mathrm{~h}$ and $0.1 \mathrm{mM}$ phenformin for $0,2,4$ and $6 \mathrm{~h}$ ), and the mTOR signaling was analyzed using western blotting with antibodies specific for p-p70 ${ }^{\mathrm{S} 6 \mathrm{~K}}, \mathrm{p} 70^{\mathrm{S} 6 \mathrm{~K}}, \mathrm{p}-4 \mathrm{E}-\mathrm{BP}-1,4 \mathrm{E}-\mathrm{BP}-1, \mathrm{p}-\mathrm{AMPK}, \mathrm{AMPK}, \mathrm{p}$-Raptor and Raptor. $\alpha$-tubulin was used as an internal control for protein loading. Each western blot is a representative result obtained from three independent experiments. AMPK, AMP-activated protein kinase.

examined cells were determined by a Seahorse Extracellular Flux XF-24 analyzer (Seahorse Bioscience, North Billerica, MA, USA) according to the manufacturer's instructions. Cells $\left(3 \times 10^{4}\right)$ were seeded in a 24 -well custom-made plate for the XF-24 analyzer. The culture medium was replaced with sodium carbonate-free DMEM ( $\mathrm{pH} 7.4)$. Prior to the assay, the cell plate and sensor cartridge were kept with $1 \mathrm{ml}$ Seahorse Bioscience XF-24 Calibrant/well in an incubator maintaining $37^{\circ} \mathrm{C}$ without $\mathrm{CO}_{2}$ overnight. The basal, protonleaked, maximal and non-OXPHOS OCRs were sequentially measured before and after the injection of $75 \mu \mathrm{l}$ of oligomycin $(2 \mu \mathrm{g} / \mathrm{ml})$, FCCP $(2 \mu \mathrm{M})$ or antimycin A $(2 \mu \mathrm{M})$, respectively. The program of the Seahorse XF-24 analyzer was set according to the manufacturer's instructions. The mitochondrial OCR was calculated by subtracting the residual rate after injection of antimycin A. The OCR and ECAR were expressed in $\mathrm{pmol} / \mathrm{min}$ and $\mathrm{mpH} / \mathrm{min}$, respectively, and normalized to the examined cell number.

Determination of intracellular ATP content. Cells $\left(2 \times 10^{5}\right)$ were seeded on 6-well plates overnight before each experiment. After treatment with vehicle (DMSO) or the mitochondrial inhibitors for $3 \mathrm{~h}$, the cells were collected and the intracellular ATP content was measured by the ATP bioluminescence assay kit (Roche Applied Science) according to the manufacturer's instructions.
Determination of lactate production. Cells $\left(2 \times 10^{5}\right)$ were seeded on 6-well plates overnight, and the culture medium was then replaced by fresh culture medium and cells were incubated for an additional $6 \mathrm{~h}$. Lactate levels in the culture medium were measured by the Lactate Assay kit (Trinity Biotech) according to the manufacturer's instructions.

Statistical analysis. Data are presented as the mean \pm SEM. Statistical differences between the control and treated groups were determined using Student's t-test, and results were considered to be a statistically significant at $\mathrm{P}<0.05$.

\section{Results}

Differential effects of the mitochondrial inhibitors and biguanide drugs on AMPK-mTOR signaling and cell survival among the HCC cell lines. To examine the effect of the mitochondrial inhibitors and biguanide drugs on mTOR signaling in different HCC cell lines, four HCC cell lines (HepG2, Mahlavu, SK-HEP-1 and HA22T/VGH cells) were treated with rotenone (a Complex I inhibitor) or oligomycin (a Complex V inhibitor). We found that the repression of mTOR signaling (indicated by the phosphorylation levels of $\mathrm{p} 70^{\mathrm{S} 6 \mathrm{~K}}$ and $4 \mathrm{E}-\mathrm{BP}-1$ ) by the mitochondrial inhibitors was only observed in the HepG2 cells (Fig. 1A, lanes 1-3) but not in the Mahlavu, SK-HEP-1 and HA22T/VGH cells (Fig. 1A, lanes 4-12). Similarly, the 
A

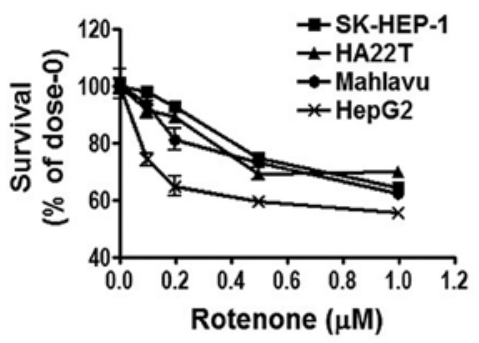

C

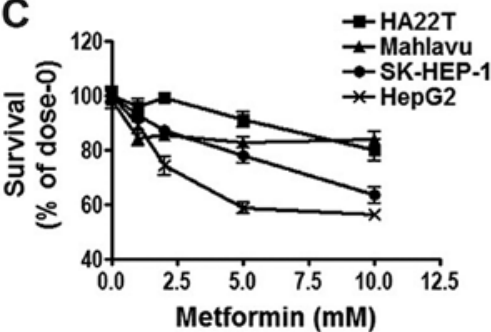

B

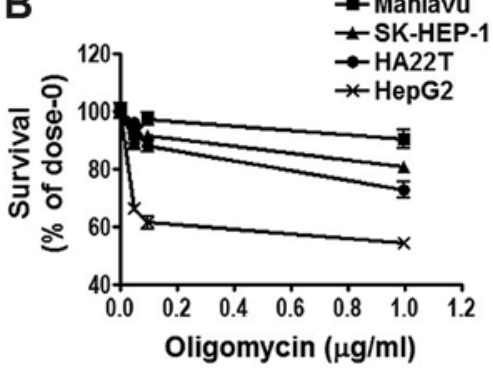

D $\quad{ }_{120}-$ Mahlavu

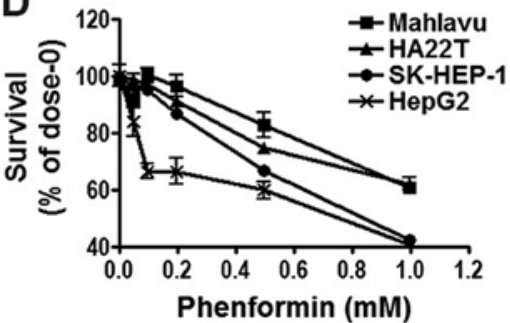

Figure 2. Effect of mitochondrial inhibitors and biguanides on HCC cell survival. HCC cell lines (HepG2, Mahlavu, SK-HEP-1 and HA22T/VGH) were treated with different doses of (A and B) mitochondrial inhibitors (rotenone and oligomycin) or (C and D) biguanide drugs (metformin and phenformin) for $24 \mathrm{~h}$, and the cytotoxicity was determined using SRB assay. HCC, hepatocellular carcinoma; SRB, sulforhodamine B.

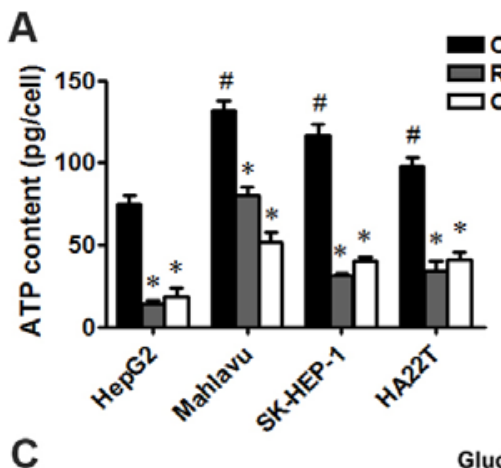

B
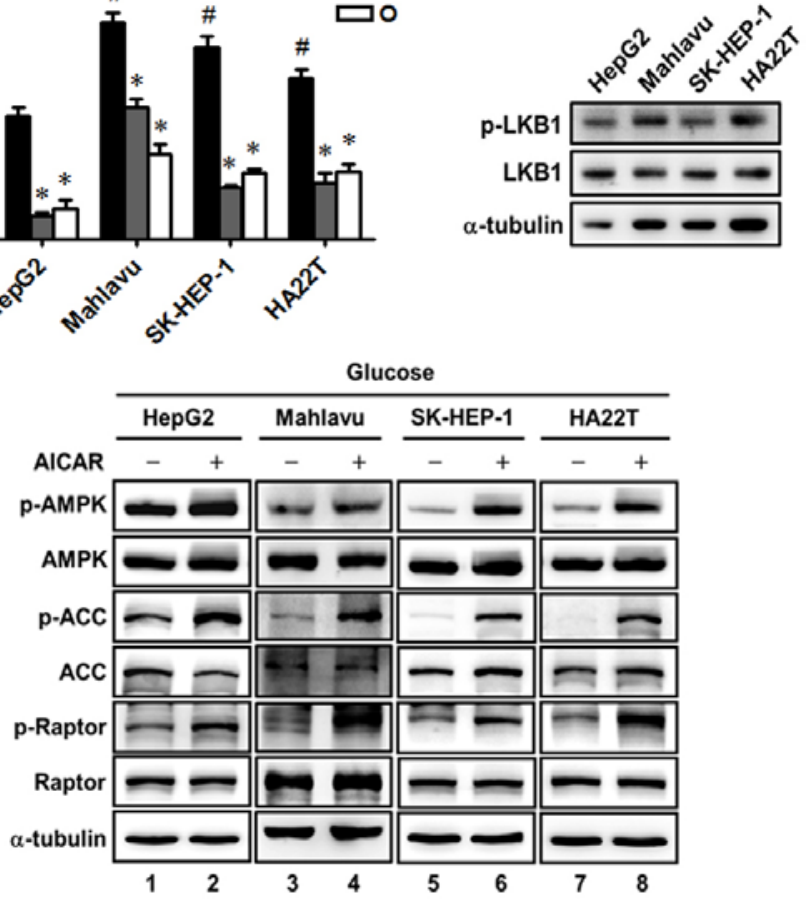

Figure 3. Effect of mitochondrial inhibitors on intracellular ATP content and the LKB-AMPK pathway in HCC cells. HCC cell lines (HepG2, Mahlavu, SK-HEP-1 and HA22T/VGH) were treated with mitochondrial inhibitors for $3 \mathrm{~h}$, and (A) cellular ATP content was analyzed using an ATP analysis kit. (B) LKB1 protein expression in the four HCC cell lines was analyzed using western blotting with an antibody against LKB1. (C) Activation of AMPK in four HCC cells was investigated using western blotting following AICAR treatment for $3 \mathrm{~h}$. Each western blot is a representative result obtained from two independent experiments $\left({ }^{*} \mathrm{P}<0.05\right.$ as compared with the vehicle group; ${ }^{\#} \mathrm{P}<0.05$ as compared with HepG2 cells). C, vehicle; $\mathrm{R}$, rotenone; $\mathrm{O}$, oligomycin; LKB, liver kinase B; AMPK, AMP-activated protein kinase; HCC, hepatocellular carcinoma.

activation of AMPK by the mitochondrial inhibitors was only observed in the HepG2 cells (Fig. 1B, lanes 1-3), but not in the other three HCC cell lines (Fig. 1B, lanes 4-12). Consistently, the activation of AMPK and inhibition of mTOR signaling by metformin and phenformin were only observed in the HepG2 cells but not in the other three HCC cell lines (Fig. 1C and D).
Due to the importance of mTOR signaling in the growth and survival of cancer cells, we next examined the effect of the mitochondria inhibitors and biguanide drugs on cell survival in the four HCC cell lines. The results revealed that the Mahlavu, SK-HEP-1 and HA22T/VGH cell lines were more resistance to the mitochondrial inhibitors (rotenone and 


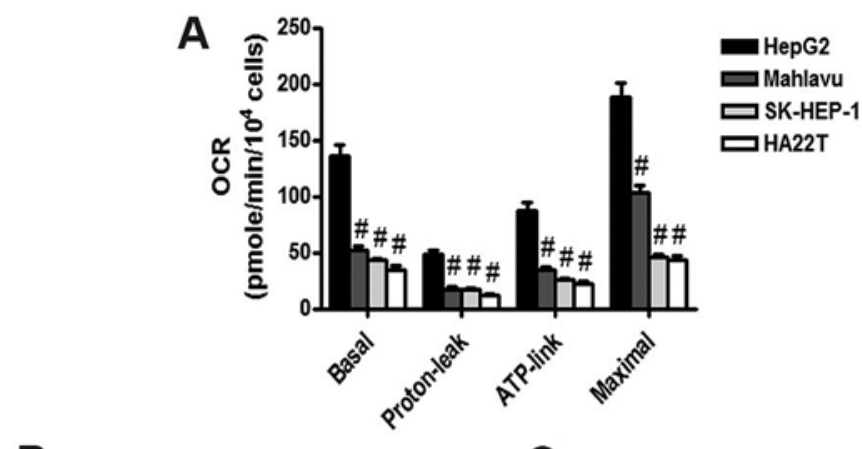

B

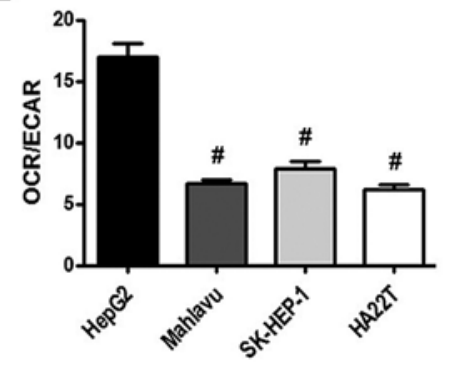

D

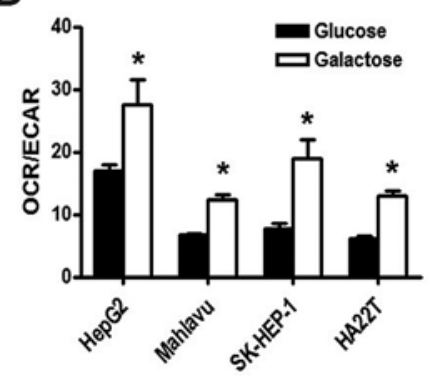

C

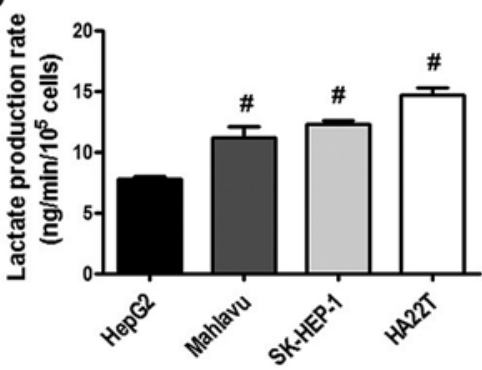

E

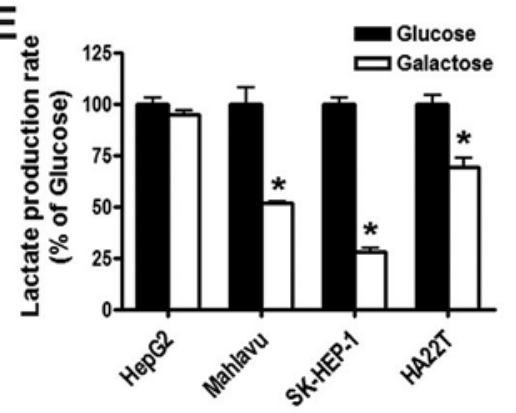

Figure 4. Energy metabolism characteristics of the HCC cells; replacement of glucose with galactose changes the energy metabolism from glycolysis to mitochondrial respiration. HCC cell lines (HepG2, Mahlavu, SK-HEP-1 and HA22T/VGH) were cultured in DMEM with $25 \mathrm{mM}$ glucose or galactose, and the parameters of energy metabolism including (A) basal OCR, proton leak, ATP-linked OCR, maximal OCR and (B and D) OCR/ECAR in the HepG2, Mahlavu, SK-HEP-1 and HA22T/VGH cell lines were analyzed using Seahorse Extracellular Flux XF analyzer. (C and E) The lactate production was detected using a lactate analysis kit ( ${ }^{\prime} \mathrm{P}<0.05$ as compared with $\mathrm{HepG} 2$ cells; ${ }^{*} \mathrm{P}<0.05$ as compared with the glucose group). HCC, hepatocellular carcinoma, OCR, oxygen consumption rate; ECAR, extracellular acidification rate.

oligomycin) (Fig. 2A and B) and biguanide drugs (metformin and phenformin) (Fig. 2C and D). These results indicated that Mahlavu, SK-HEP-1 and HA22T/VGH cells were more resistant to mitochondrial inhibitors and biguanide drugs as compared with the HepG2 cells.

HCC cells with higher glycolysis activity are more resistant to mitochondrial inhibitors and biguanide drugs. We further determined the intracellular ATP levels and found that the ATP level in the HepG2 cells was lower than levels in the Mahlavu, SK-HEP-1 and HA22T/VGH cells (Fig. 3A), and the mitochondrial inhibitors markedly decreased the intracellular ATP to a similar extent in the four HCC cell lines (Fig. 3A). Moreover, all the four HCC cells were found to express LKB1 protein and similar levels of phosphorylated LKB1, indicating that LKB1 was not deficient in the four HCC cell lines (Fig. 3B). In addition, we treated the four HCC cell lines with an AMPK activator AICAR, and found that AICAR significantly increased the phosphorylation of AMPK and the AMPK downstream proteins, such as acetyl-CoA carboxylase (ACC) and Raptor, in the four HCC cell lines (Fig. 3C). These results revealed that there were no significant differences in the ATP changes in response to mitochondrial inhibitors, LKB1 protein expression and AMPK function among the four HCC cells. Therefore, these factors did not play a major role in the resistance of the Mahlavu, SK-HEP-1 and HA22T/VGH cells to mitochondrial inhibitors and biguanide.

We next determined the energy metabolism in the HCC cell lines using the Seahorse Extracellular Flux XF-24 analyzer. We found that mitochondrial OCR including the basal, protonleaked, ATP-link and maximal OCRs in the HepG2 cells were higher than those in the Mahlavu, SK-HEP-1 and HA22T/VGH cells (Fig. 4A). Moreover, the ratio of OCR/ECAR (Fig. 4B) in HepG2 cells was higher than that in the Mahlavu, SK-HEP-1 and HA22T/VGH cells. In addition, the lactate production rate in HepG2 cells was lower than that in the Mahlavu, SK-HEP-1 and HA22T/VGH cells (Fig. 4C). These results indicated that HepG2 cells have higher OXPHOS activity; while Mahlavu, SK-HEP-1 and HA22T/VGH cells have higher glycolysis activity. These results suggest that HCC cells with high glycolysis activity are more resistant to mitochondrial inhibitors and biguanide drugs. 


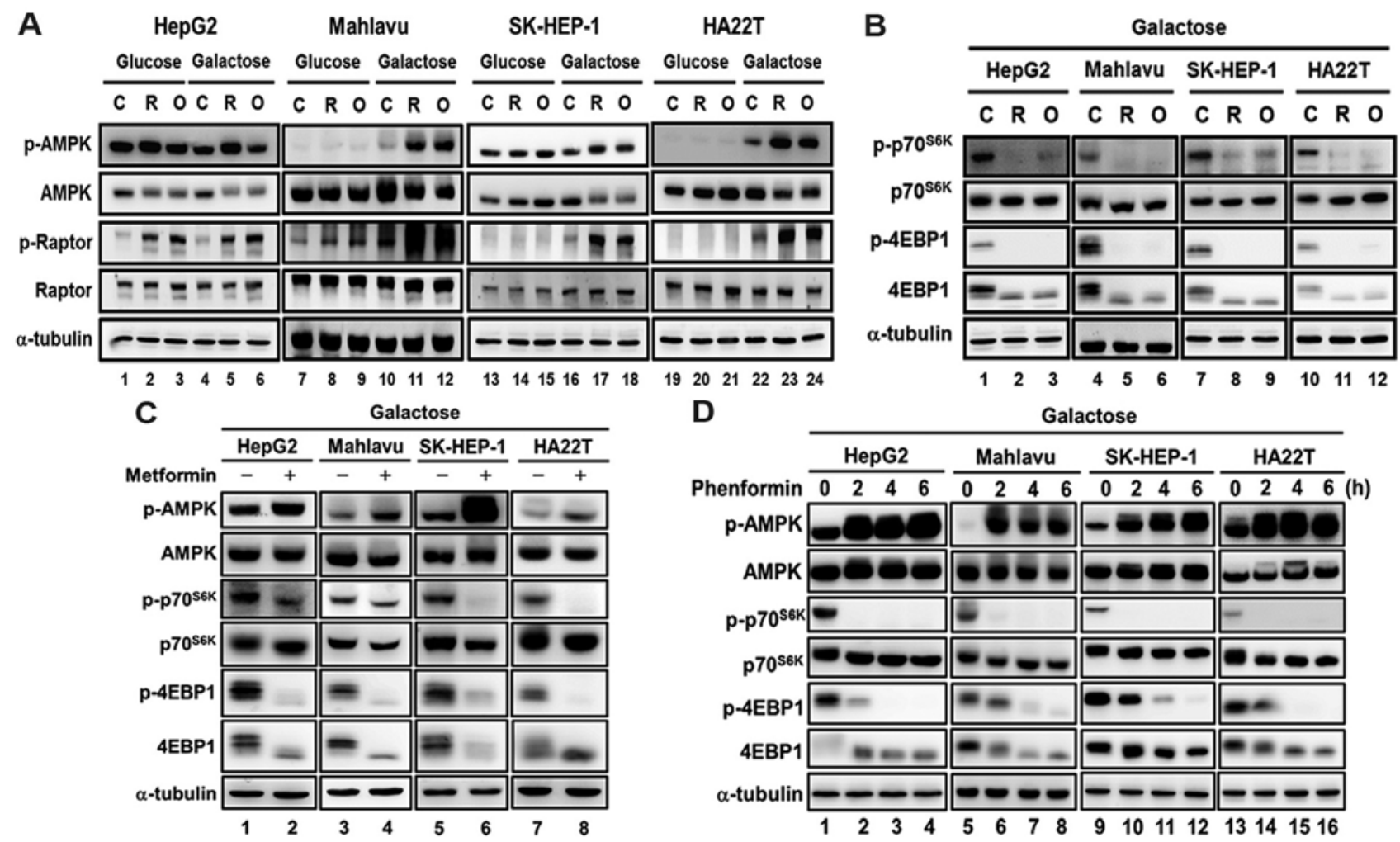

Figure 5. Replacing glucose with galactose increases the response to mitochondrial inhibitors and biguanides in regards to AMPK-mTOR signaling in HCC cells. HCC cell lines (HepG2, Mahlavu, SK-HEP-1 and HA22T/VGH) were cultured in DMEM with $25 \mathrm{mM}$ glucose or galactose, and then treated with (A and B) mitochondrial inhibitors $(0.2 \mu \mathrm{M}$ rotenone $(\mathrm{R})$ and $1 \mu \mathrm{g} / \mathrm{ml}$ oligomycin $(\mathrm{O})]$ for $3 \mathrm{~h}$ or $(\mathrm{C}$ and D) biguanide drugs $(5 \mathrm{mM}$ metformin for $9 \mathrm{~h}$, and $0.1 \mathrm{mM}$ phenformin for $0,2,4$ and $6 \mathrm{~h}$ ), and the AMPK and mTOR signaling was analyzed using western blotting with antibodies specific for p-p70 ${ }^{\mathrm{s}}, \mathrm{K}$, $70^{\mathrm{S} 6 \mathrm{~K}}, \mathrm{p}-4 \mathrm{E}-\mathrm{BP}-1$, 4E-BP-1, p-AMPK, AMPK, p-Raptor and Raptor. $\alpha$-tubulin was used as an internal control for protein loading. Each western blot shown is a representative result obtained from three independent experiments. AMPK, AMP-activated protein kinase; HCC, hepatocellular carcinoma.

Increased OCR/ECAR by galactose medium enhances the cell sensitivity to mitochondrial inhibitors and biguanide drugs. To examine whether energy metabolism determines the response to mitochondrial inhibitors, we replaced glucose with galactose in the culture medium. The results revealed that the galactose medium increased the OCR/ECAR ratio of the HCC cells (Fig. 4D), and decreased the lactate production rate (Fig. 4E) as compared with parameters in the HCC cells grown in the glucose medium. Parental HepG2 cells have higher OXPHOS activity; as a result, the lactate production rate did not show a significant change between the glucose and galactose medium in the HepG2 cells (Fig. 4E). These results indicate that the galactose medium altered the energy metabolism to enhance mitochondrial respiration in the HCC cells.

We further evaluated whether the change in energy metabolism alters the effect of mitochondrial inhibitors and biguanides on AMPK-mTOR signaling and cell survival. We found that the extent of AMPK activation (Fig. 5A, C and D), repression of mTOR signaling (Fig. 5B-D) and the cytotoxicity (Fig. 6) were significantly increased in the HCC cells when they were grown in the galactose medium containing mitochondrial inhibitors and biguanides. These results together suggest that the increase in the OCR/ECAR ratio enhances the cell sensitivity to mitochondrial inhibitors and biguanide drugs.

Decreases in cyclin DI and Mcl-1 are associated with the cytotoxicity in response to mitochondrial inhibitors and bigu- anide drugs. Due to the importance of cyclin D1 and Mcl-1 for cell cycle progression and cell survival in cancer cells, we further investigated the effect of mitochondrial inhibitors and phenformin on the levels of cyclin D1 and Mcl-1 in the HCC cells grown in glucose or galactose medium. We found that downregulation of cyclin D1 and Mcl-1 expression by mitochondrial inhibitors (Fig. 7A and B, lanes 1-3) and phenformin (Fig. 7C, lane 1-4; Fig. 7D, lane 1 and 2) were observed only in HepG2 cells but not in the Mahlavu, SK-HEP-1 and HA22T/VGH cell lines cultured in glucose medium. In the galactose medium, mitochondrial inhibitors and phenformin significantly decreased the protein expression of cyclin D1 and Mcl-1 in the four HCC cell lines (Fig. 7A and B, lanes 4-6; Fig. 7C, lanes 5-8; Fig. 7D, lanes 3 and 4). These results suggest that the downregulation of cyclin D1 and Mcl-1 are associated with the cytotoxicity induced by mitochondrial inhibitors and biguanide drugs.

\section{Discussion}

In the present study, we demonstrated that changing energy metabolism from glycolysis to mitochondrial respiration enhances the sensitivity of HCC cell lines to mitochondrial inhibitors and biguanide drugs. HepG2 cells had a higher mitochondrial respiration rate (OCR), lower glycolysis (ECAR) and lower lactate production rate, and were found to be more sensitive to mitochondrial inhibitors, suggesting that the energy metabolism of the cells is more dependent 

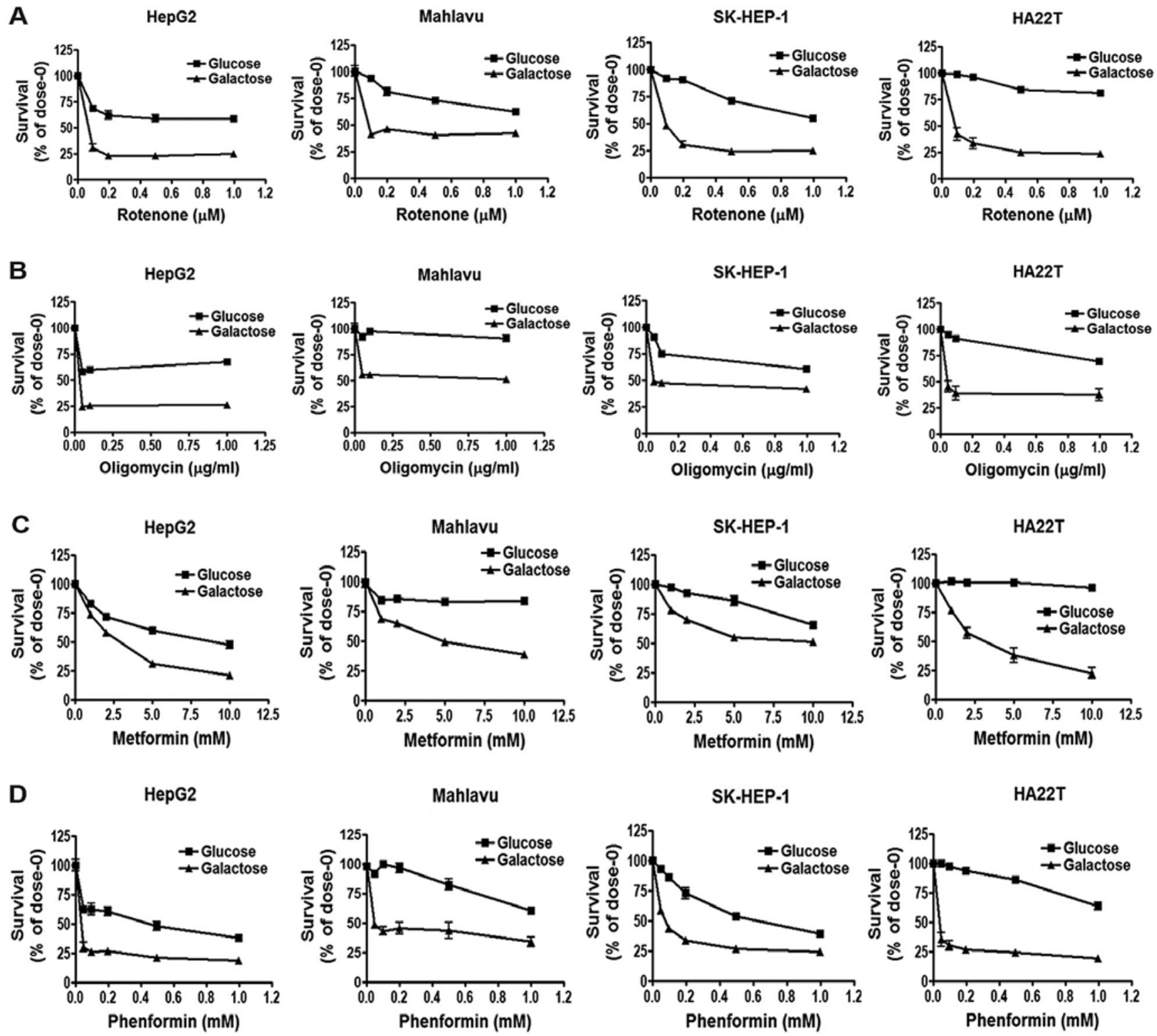

Figure 6. Replacing glucose with galactose sensitizes the HCC cell lines to mitochondrial inhibitors and biguanides. HCC cell lines (HepG2, Mahlavu, SK-HEP-1 and HA22T/VGH) were cultured in DMEM medium with $25 \mathrm{mM}$ glucose or galactose, and then treated with (A and B) mitochondrial inhibitors (rotenone and oligomycin) or (C and D) biguanide drugs (metformin and phenformin) at different doses for $24 \mathrm{~h}$, and the cytotoxicity was analyzed using the SRB assay. HCC, hepatocellular carcinoma; SRB, sulforhodamine B.

on mitochondrial OXPHOS. In contrast, the Mahlavu, SK-HEP-1 and HA22T/VGH cell lines had higher glycolysis, lower mitochondrial respiration and were more resistant to mitochondrial inhibitors, indicating that their metabolism was more dependent on glycolysis. Thus, high glycolysis activity may render HCC cells more resistant to biguanide drugs. In addition, we found that a change in energy metabolism from glycolysis to OXPHOS sensitized the HCC cells to mitochondrial inhibitors and biguanide drugs. These results suggest that energy metabolism plays an important role in regulating the sensitivity of $\mathrm{HCC}$ cells to biguanide drugs.

The biguanide drugs were reported to inhibit mitochondrial respiratory chain Complex I (29) and activate AMPK (33). Accumulating evidence indicates that the biguanide drugs reduce the risk of breast cancer $(34,35)$, HCC (25-27) and pancreatic cancer $(36,37)$, and thus these drugs have been proposed as adjuvant reagents for cancer therapy $(28,29)$.
Recent studies have also revealed that OXPHOS plays an essential role in tumor initiation and metastasis $(38,39)$. These findings suggest that the OXPHOS can be therapeutically targeted in cancers. Our present results revealed that HCC cells (for example, HepG2 cells) exhibiting higher OXPHOS activity were more sensitive to biguanide drugs, which further suggest that the biguanide drugs may be potential agents to inhibit cancer metastasis and progression.

Cancer cells usually exhibit various energy metabolism characteristics and have different responses to therapeutic agents. To evaluate whether cellular energy metabolism regulates the sensitivity to biguanide drugs of HCC cells, we used mitochondrial inhibitors to reduce the intracellular ATP content in the HCC cell lines (Fig. 3A), and found that HepG2 cells were more sensitive than the Mahlavu, SK-HEP-1 and HA22T/VGH cells. Moreover, the activation of AMPK was detected only in HepG2 cells rather than the other HCC cell 
A

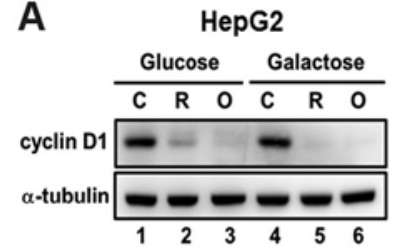

B

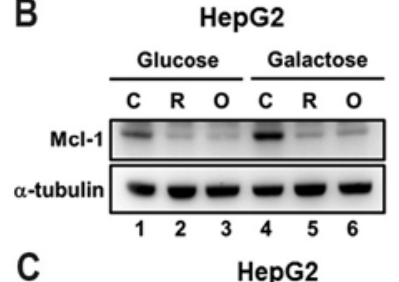

C

HepG2

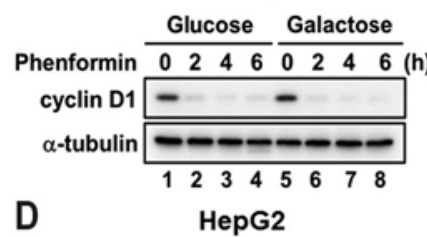

D

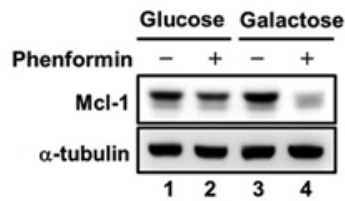

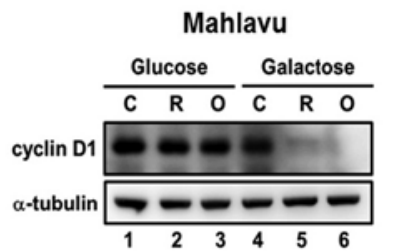

Mahlavu

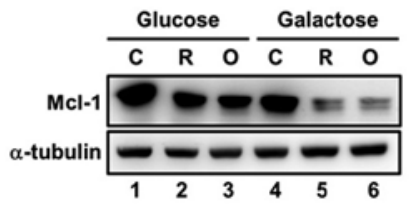

Mahlavu

Glucose Galactose

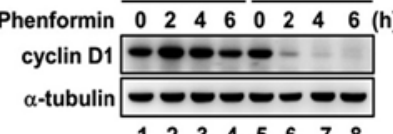

$\begin{array}{lllllll}2 & 3 & 4 & 5 & 6 & 7 & 8\end{array}$

Mahlavu

Glucose Galactose

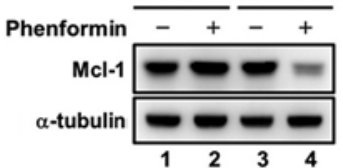

SK-HEP-1

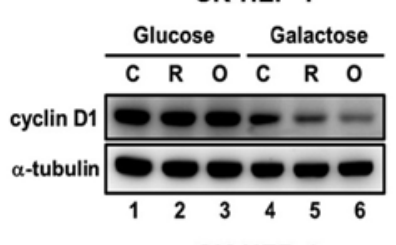

SK-HEP-1

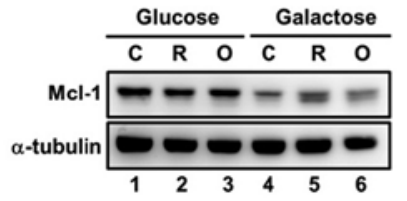

SK-HEP-1

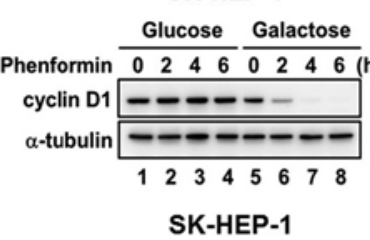

Glucose Galactose

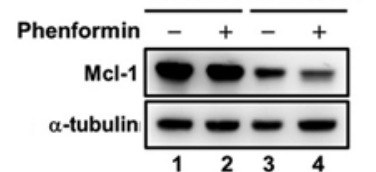

HA22T

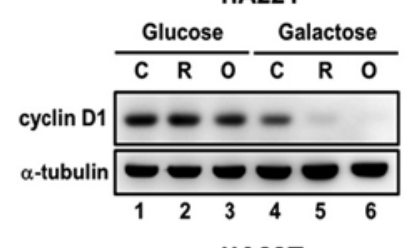

HA22T

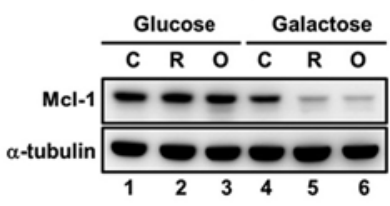

HA22T

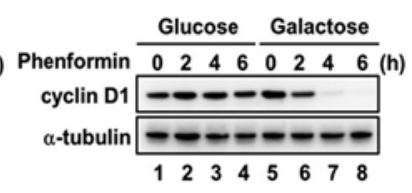

HA22T

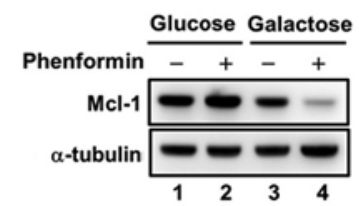

Figure 7. Downregulation of cyclin D1 and Mcl-1 are associated with the cytotoxicity induced by mitochondrial inhibitors and biguanides. HCC cell lines (HepG2, Mahlavu, SK-HEP-1 and HA22T/VGH) were cultured in DMEM medium with 25 mM glucose or galactose, then treated with mitochondrial inhibitors (A and B) $(0.2 \mu \mathrm{M}$ rotenone or $1 \mu \mathrm{g} / \mathrm{ml}$ oligomycin for $4 \mathrm{~h})$ or $(\mathrm{C}$ and $\mathrm{D}) 0.1 \mathrm{mM}$ phenformin for $0,2,4$ and $6 \mathrm{~h}$, the protein expressions of cyclin $\mathrm{D} 1$ and Mcl-1 were analyzed using western blotting with antibodies specific for cyclin D1 and Mcl-1. The $\alpha$-tubulin was used as an internal control for protein loading. The western blotting is a representative result obtained from two independent experiments. HCC, hepatocellular carcinoma.

lines (Mahlavu, SK-HEP-1 and HA22T/VGH cells) (Fig. 1B). The lower response of AMPK to the mitochondrial inhibitors in the Mahlavu, SK-HEP-1 and HA22T/VGH cells was associated with their higher glycolysis and lower mitochondrial respiration rate. Moreover, we altered the cellular energy metabolism from glycolysis to OXPHOS in the Mahlavu, SK-HEP-1 and HA22T/VGH cells, and found that AMPK was activated by these treatments (Fig. 5A, C and D). These results indicated that the cell sensitivity and the activation of AMPK in response to mitochondrial inhibitors or biguanide drugs are associated with cellular energy metabolism. Further investigation is warranted to ascertain whether the change in energy metabolism from glycolysis to OXPHOS alters the sensitivity in other types of cancer such as breast and pancreatic cancers.

In the present study, galactose was used for altering the cellular metabolism from glycolysis to mitochondrial OXPHOS and this enhanced cell sensitivity to the biguanides. This combination of galactose with biguanide drugs may provide a beneficial strategy for TACE in HCC patients. Recent progress in understanding the mechanisms for exhibiting the Warburg effect in cancer cells has identified several proteins, including lactate dehydrogenase A (LDHA), pyruvate kinase M2 isoform (PKM2) and pyruvate dehydrogenase kinases (PDKs) (40-42). Many proliferating cells or cancer cells express a high level of LDHA resulting in glycolytic flux toward lactate production even in the presence of oxygen, and inhibition of LDHA was found to induce the oxygen consumption rate and inhibit tumor growth $(41,43)$. In addition, it has been reported that PKM2 is highly expressed in several types of cancer including breast, cervical, colon, gastrointestinal, hepatoma and lung cancer (44), and knockdown of PKM2 in several types of cancer was found to lead to increased OCR, decreased lactate production and inhibition of tumor growth (45). Moreover, PDKs are upregulated in cancer cells due to high expression of HIF-1 $\alpha$, and activated PDKs inhibit pyruvate dehydrogenases, which convert pyruvate into acetyl-CoA for tricarboxylic acid cycle, and thereby switch the energy metabolism from OXPHOS to aerobic glycolysis (40). Therefore, further investigation is warranted to ascertain whether inhibition of these enzymes (LDHA, PKM2 or PDKs) alters the energy metabolism in HCC cells and sensitizes HCC cells to mitochondrial inhibitors or biguanide drugs.

In conclusion, we found that $\mathrm{HCC}$ cells which exhibit higher glycolysis and lower mitochondrial respiration are more resistant to mitochondrial inhibitors and biguanide drugs. Our findings also provide evidence to suggest that altering the energy metabolism from aerobic glycolysis to OXPHOS enhances the cell sensitivity to biguanides. These findings suggest that a change in energy metabolism from glycolysis to OXPHOS enhances the effect of biguanide drugs in HCC therapy.

\section{Acknowledgements}

We thank Ms. Shu-Hui Li for the excellent technical assistance. This study was partly supported by a grant from the Center of Excellence for Cancer Research at Taipei Veterans General Hospital, the Ministry of Health 
and Welfare, Executive Yuan (MOHW103-TD-B-111-02; MOHW104-TDU-B-211-124-001), a grant from the Ministry of Education, Aim for the Top University Plan, and the grant MOST101-2320-B-010-068-MY3 from the Ministry of Science and Technology, Taiwan.

\section{References}

1. Belghiti J and Kianmanesh R: Surgical treatment of hepatocellular carcinoma. HPB Oxf 7: 42-49, 2005.

2. Marin JJ, Castaño B, Martinez-Becerra P, Rosales R and Monte MJ: Chemotherapy in the treatment of primary liver tumours. Cancer Ther 6: 711-728, 2008.

3. Wang Y and Shen Y: Unresectable hepatocellular carcinoma treated with transarterial chemoembolization: Clinical data from a single teaching hospital. Int J Clin Exp Med 6: 367-371, 2013.

4. Forner A, Llovet JM and Bruix J: Hepatocellular carcinoma. Lancet 379: 1245-1255, 2012

5. Vander Heiden MG, Cantley LC and Thompson CB: Understanding the Warburg effect: The metabolic requirements of cell proliferation. Science 324: 1029-1033, 2009.

6. Hanahan D and Weinberg RA: Hallmarks of cancer: The next generation. Cell 144: 646-674, 2011.

7. Chen Z, Lu W, Garcia-Prieto C and Huang P: The Warburg effect and its cancer therapeutic implications. J Bioenerg Biomembr 39 267-274, 2007.

8. Hsu CC, Lee HC and Wei YH: Mitochondrial DNA alterations and mitochondrial dysfunction in the progression of hepatocellular carcinoma. World J Gastroenterol 19: 8880-8886, 2013.

9. Lee HC and Wei YH: Mitochondrial DNA instability and metabolic shift in human cancers. Int J Mol Sci 10: 674-701, 2009.

10. DeBerardinis RJ, Lum JJ, Hatzivassiliou G and Thompson CB: The biology of cancer: Metabolic reprogramming fuels cell growth and proliferation. Cell Metab 7: 11-20, 2008.

11. Tennant DA, Durán RV and Gottlieb E: Targeting metabolic transformation for cancer therapy. Nat Rev Cancer 10: 267-277, 2010.

12. Ma XM and Blenis J: Molecular mechanisms of mTOR-mediated translational control. Nat Rev Mol Cell Biol 10: 307-318, 2009.

13. Shimobayashi M and Hall MN: Making new contacts: The mTOR network in metabolism and signalling crosstalk. Nat Rev Mol Cell Biol 15: 155-162, 2014.

14. Engelman JA, Luo J and Cantley LC: The evolution of phosphatidylinositol 3-kinases as regulators of growth and metabolism. Nat Rev Genet 7: 606-619, 2006.

15. Yuan TL and Cantley LC: PI3K pathway alterations in cancer: Variations on a theme. Oncogene 27: 5497-5510, 2008.

16. Vivanco I and Sawyers CL: The phosphatidylinositol 3-kinase AKT pathway in human cancer. Nat Rev Cancer 2: 489-501, 2002 .

17. Gwinn DM, Shackelford DB, Egan DF, Mihaylova MM, Mery A, Vasquez DS, Turk BE and Shaw RJ: AMPK phosphorylation of raptor mediates a metabolic checkpoint. Mol Cell 30: 214-226, 2008.

18. Hardie DG, Ross FA and Hawley SA: AMPK: A nutrient and energy sensor that maintains energy homeostasis. Nat Rev Mol Cell Biol 13: 251-262, 2012

19. Fernandez P, Carretero J, Medina PP, Jimenez AI, RodriguezPerales S, Paz MF, Cigudosa JC, Esteller M, Lombardia L, Morente M, et al: Distinctive gene expression of human lung adenocarcinomas carrying LKB1 mutations. Oncogene 23: 5084-5091, 2004.

20. Huang YH, Chen ZK, Huang KT, Li P, He B, Guo X, Zhong JQ, Zhang QY, Shi HQ, Song QT, et al: Decreased expression of LKB1 correlates with poor prognosis in hepatocellular carcinoma patients undergoing hepatectomy. Asian Pac J Cancer Prev 14: 1985-1988, 2013.

21. Sahin F, Maitra A, Argani P, Sato N, Maehara N, Montgomery E, Goggins M, Hruban RH and Su GH: Loss of Stk11/Lkb1 expression in pancreatic and biliary neoplasms. Mod Pathol 16 : 686-691, 2003

22. Shen Z, Wen XF, Lan F, Shen ZZ and Shao ZM: The tumor suppressor gene $L K B 1$ is associated with prognosis in human breast carcinoma. Clin Cancer Res 8: 2085-2090, 2002.

23. Hezel AF and Bardeesy N: LKB1; linking cell structure and tumor suppression. Oncogene 27: 6908-6919, 2008.

24. Bailey CJ and Turner RC: Metformin. N Engl J Med 334: 574-579, 1996.
25. Chen HP, Shieh JJ, Chang CC, Chen TT, Lin JT, Wu MS, Lin JH and Wu CY: Metformin decreases hepatocellular carcinoma risk in a dose-dependent manner: Population-based and in vitro studies. Gut 62: 606-615, 2013

26. Hassan MM, Curley SA, Li D, Kaseb A, Davila M, Abdalla EK Javle M, Moghazy DM, Lozano RD, Abbruzzese JL, et al: Association of diabetes duration and diabetes treatment with the risk of hepatocellular carcinoma. Cancer 116: 1938-1946, 2010.

27. Libby G, Donnelly LA, Donnan PT, Alessi DR, Morris AD and Evans JM: New users of metformin are at low risk of incident cancer: A cohort study among people with type 2 diabetes. Diabetes Care 32: 1620-1625, 2009.

28. Petrushev B, Tomuleasa C, Soritau O, Aldea M, Pop T, Susman S, Kacso G, Berindan I, Irimie A and Cristea V: Metformin plus PIAF combination chemotherapy for hepatocellular carcinoma. Exp Oncol 34: 17-24, 2012.

29. Pollak M: Potential applications for biguanides in oncology. J Clin Invest 123: 3693-3700, 2013.

30. Dowling RJ, Zakikhani M, Fantus IG, Pollak M and Sonenberg N: Metformin inhibits mammalian target of rapamycin-dependent translation initiation in breast cancer cells. Cancer Res 67: 10804-10812, 2007.

31. Kalender A, Selvaraj A, Kim SY, Gulati P, Brûlé S, Viollet B, Kemp BE, Bardeesy N, Dennis P, Schlager JJ, et al: Metformin, independent of AMPK, inhibits mTORC1 in a rag GTPasedependent manner. Cell Metab 11: 390-401, 2010.

32. Hsu CC, Wang CH, Wu LC, Hsia CY, Chi CW, Yin PH, Chang CJ, Sung MT, Wei YH, Lu SH, et al: Mitochondrial dysfunction represses HIF-1 $\alpha$ protein synthesis through AMPK activation in human hepatoma HepG2 cells. Biochim Biophys Acta 1830: 4743-4751, 2013

33. Stephenne X, Foretz M, Taleux N, van der Zon GC, Sokal E, Hue L, Viollet B and Guigas B: Metformin activates AMP-activated protein kinase in primary human hepatocytes by decreasing cellular energy status. Diabetologia 54: 3101-3110, 2011.

34. Bodmer M, Meier C, Krähenbühl S, Jick SS and Meier CR: Long-term metformin use is associated with decreased risk of breast cancer. Diabetes Care 33: 1304-1308, 2010.

35. Chlebowski RT, McTiernan A, Wactawski-Wende J, Manson JE, Aragaki AK, Rohan T, Ipp E, Kaklamani VG, Vitolins M, Wallace R, et al: Diabetes, metformin, and breast cancer in postmenopausal women. J Clin Oncol 30: 2844-2852, 2012.

36. Bodmer M, Becker C, Meier C, Jick SS and Meier CR: Use of antidiabetic agents and the risk of pancreatic cancer: A casecontrol analysis. Am J Gastroenterol 107: 620-626, 2012.

37. Li D, Yeung SC, Hassan MM, Konopleva M and Abbruzzese JL: Antidiabetic therapies affect risk of pancreatic cancer. Gastroenterology 137: 482-488, 2009.

38. LeBleu VS, O'Connell JT, Gonzalez Herrera KN, Wikman H, Pantel K, Haigis MC, de Carvalho FM, Damascena A, Domingos Chinen LT, Rocha RM, et al: PGC- $1 \alpha$ mediates mitochondrial biogenesis and oxidative phosphorylation in cancer cells to promote metastasis. Nat Cell Biol 16: 992-1003, 1001-1015, 2014.

39. Tan AS, Baty JW, Dong LF, Bezawork-Geleta A, Endaya B, Goodwin J, Bajzikova M, Kovarova J, Peterka M, Yan B, et al: Mitochondrial genome acquisition restores respiratory function and tumorigenic potential of cancer cells without mitochondrial DNA. Cell Metab 21: 81-94, 2015.

40. Cairns RA, Harris IS and Mak TW: Regulation of cancer cell metabolism. Nat Rev Cancer 11: 85-95, 2011.

41. Lunt SY and Vander Heiden MG: Aerobic glycolysis: Meeting the metabolic requirements of cell proliferation. Annu Rev Cell Dev Biol 27: 441-464, 2011.

42. Vander Heiden MG, Christofk HR, Schuman E, Subtelny AO, Sharfi H, Harlow EE, Xian J and Cantley LC: Identification of small molecule inhibitors of pyruvate kinase M2. Biochem Pharmacol 79: 1118-1124, 2010.

43. Le A, Cooper CR, Gouw AM, Dinavahi R, Maitra A, Deck LM, Royer RE, Vander Jagt DL, Semenza GL and Dang CV: Inhibition of lactate dehydrogenase A induces oxidative stress and inhibits tumor progression. Proc Natl Acad Sci USA 107: 2037-2042, 2010.

44. Wong N, De Melo J and Tang D: PKM2, a central point of regulation in cancer metabolism. Int J Cell Biol 2013: 242513, 2013.

45. Christofk HR, Vander Heiden MG, Harris MH, Ramanathan A, Gerszten RE, Wei R, Fleming MD, Schreiber SL and Cantley LC: The M2 splice isoform of pyruvate kinase is important for cancer metabolism and tumour growth. Nature 452: 230-233, 2008. 